\title{
COMPARATIVE STUDY BETWEEN PRE-SEASONAL AND INTRA-SEASONAL TREATMENT OF ALLERGIC RHINITIS USING A NEW 808NM DIODE LASER SYSTEM
}

\author{
Katsumi Sasaki ${ }^{1}$,Toshio Ohshiro ${ }^{1,2}$,Takafumi Ohshiro ${ }^{1}$, \\ Sho Kanzaki ${ }^{3}$, Kaoru Ogawa ${ }^{3}$ \\ 1: Obshiro Clinic, Shinanomachi, Tokyo, Japan, and \\ 2: Japan Medical Laser Laboratory, Shinanomachi, Tokyo, Japan, and \\ 3: Department of ENT, Keio University School of Medicine, Shinanomachi, Tokyo, Japan.
}

\begin{abstract}
Background and Aims: Allergic rhinitis annually reaches epidemic proportions in Japan, and represents a major problem. The application of the $\mathrm{CO} 2$ laser in the treatment of allergic rhinitis was first successfully reported in 1982, but $\mathrm{CO}_{2}$ laser ablation is associated with side effects. The Ohshiro Clinic started using the conventional Nd: YAG laser for the treatment of allergic rhinitis in 1993, and from 2005 we started using a diode laser-pumped Nd: YAG laser with efficacy and safety at least equal to the others. Even more recently, we have adopted a novel $808 \mathrm{~nm}$ diode laser, and the present retrospective study examined the difference in of the efficacy rate between the preseasonal and intra-seasonal treatment of allergic rhinitis with this system.

Subjects and methods: One thousand, two hundred and ten patients primarily presented to the Ohshiro Clinic with allergic rhinitis during the period from December 2010 to April 2011, of whom 531 were selected for blood tests to identify 13 kinds of Japanese allergens including cedar pollen. In the 2010-2011 season, huge clouds of cedar pollen appeared from February $26^{\text {th }}$. The subjects were therefore divided into two groups depending on the treatment period, namely the preseasonal and intra-seasonal groups, December $1^{\text {st }}, 2010$ to February $25^{\text {th }}, 2011$ and February $26^{\text {th }}$ to April $6^{\text {th }} 2011$, respectively. All patients were treated with the new $808 \mathrm{~nm}$ diode laser system. Results: A high success rate was achieved in both groups, with no significant difference seen regarding the symptoms of rhinorrhea, obstruction, sneezing, quality of life (QOL) or severity.

Conclusions: The $808 \mathrm{~nm}$ diode laser offers an excellent solution for the uncomfortable symptoms of allergic rhinitis, and could be equally well applied before or during the Japanese cedar pollen dispersed season.
\end{abstract}

Key words: Cedar pollenosis, diode laser, immunoglobulin E, RAST test, type 1 allergic reaction, anti-histamine

\section{Introduction}

In Japan, 15-20\% of the population has been reported annually as suffering from pollinosis, the most typical seasonal allergic rhinitis the spring season (midFebruary to late-April) caused by the Japanese cedar

\section{Addressee for Correspondence:}

Katsumi Sasaki MD,

Ohshiro Clinic,

$2^{\text {nd }}$ Floor, JR Shinanomachi Station Building,

Shinaomachi 34, Shinjuku-ku, Tokyo,Japan 160-0016

Email: sasaki@ohshiro.com tree (Cryptomeria japonica). Allergic rhinitis is a Type 1 allergic reaction affecting the nasal mucous membrane. Symptoms consist of repetitive attacks of sneezing, rhinorrhea, and nasal obstruction. The most severe symptom is the inability to manage normal daily activities with a poor quality of life (QOL), and the severity of symptoms tends to increase in most patients. The number of patients with really severe symptoms is growing annually. The usual therapy for pollinosis in

Manuscript received: August 6th, 2012

Accepted for publication: September 12th, 2012 
the ENT clinic is prescription of oral anti-histamines and topical nasal decongestants with steroids. The side effects of the medications are drowsiness and dryness of the throat which can disturb the QOL almost as much as the symptoms they are trying to alleviate. Recently the usual protocol for these medications is to start patients on them 2-3 weeks before the onset of the symptoms. Oral ingestion must be continued through the spring season. Also the patients must protect themselves with masks and goggles to prevent exposure to high pollen counts.

Laser ablation of the mucosal lining of the inferior turbinate using $\mathrm{CO} 2$ laser was first reported by Mittleman in 1982, 1) and showed promising results ranging from 60 to $85 \%$ efficacy in prevention of onset of nasal symptom. However $\mathrm{CO} 2$ laser ablation has certain severe side effects. Therefore the Ohshiro Clinic started using the flash lamp pumped Nd: YAG laser for the treatment of allergic rhinitis in 1993. From 2005, we started using a diode laser-pumped Nd: YAG laser manufactured by Fibertech Co. We showed that this system was superior compared to the $\mathrm{CO} 2$ laser and the conventional flash lump pumped system, with comparative efficacy and safety. ${ }^{2)}$ Even more recently, we have adopted a new $808 \mathrm{~nm}$ diode laser system for treatment of allergic rhinitis, and in the present retro- spective study we examined and assessed the difference in the efficacy rate between pre-seasonal and intra-seasonal treatment with this new system.

\section{Patients and Methods}

One thousand, two hundred and ten patients primarily presented to the Ohshiro Clinic with allergic rhinitis during the period of December 2010 to April 2011. Five hundred and thirty-one patients were selected to undergo blood tests to identify 13 kinds of allergen including cedar pollen. In the 2010-2011 seasons, large clouds of Japanese cedar pollen appeared in the air from February $26^{\text {th }}$. The subjects were therefore divided into two groups were depending on the treatment period. Patients in the pre-seasonal group were treated from December $1^{\text {st }} 2010$ to February $2^{\text {th }} 2011$. In this group there were 316 patients consisting of 168 males and 148 females, and the average age was 40.7 years. The other group of patients, the intra-seasonal group, was treated from February $26^{\text {th }}$ to April $6^{\text {th }} 2011$. There were 225 patients consisting of 116 males and 109 females, and the average age was 39.32 years.

All patients were treated with the new $808 \mathrm{~nm}$ Diode laser, DVL-20B (ASUKA Medical Co., Tokyo, Japan) at an output power of $8 \mathrm{~W}$, and a total energy of

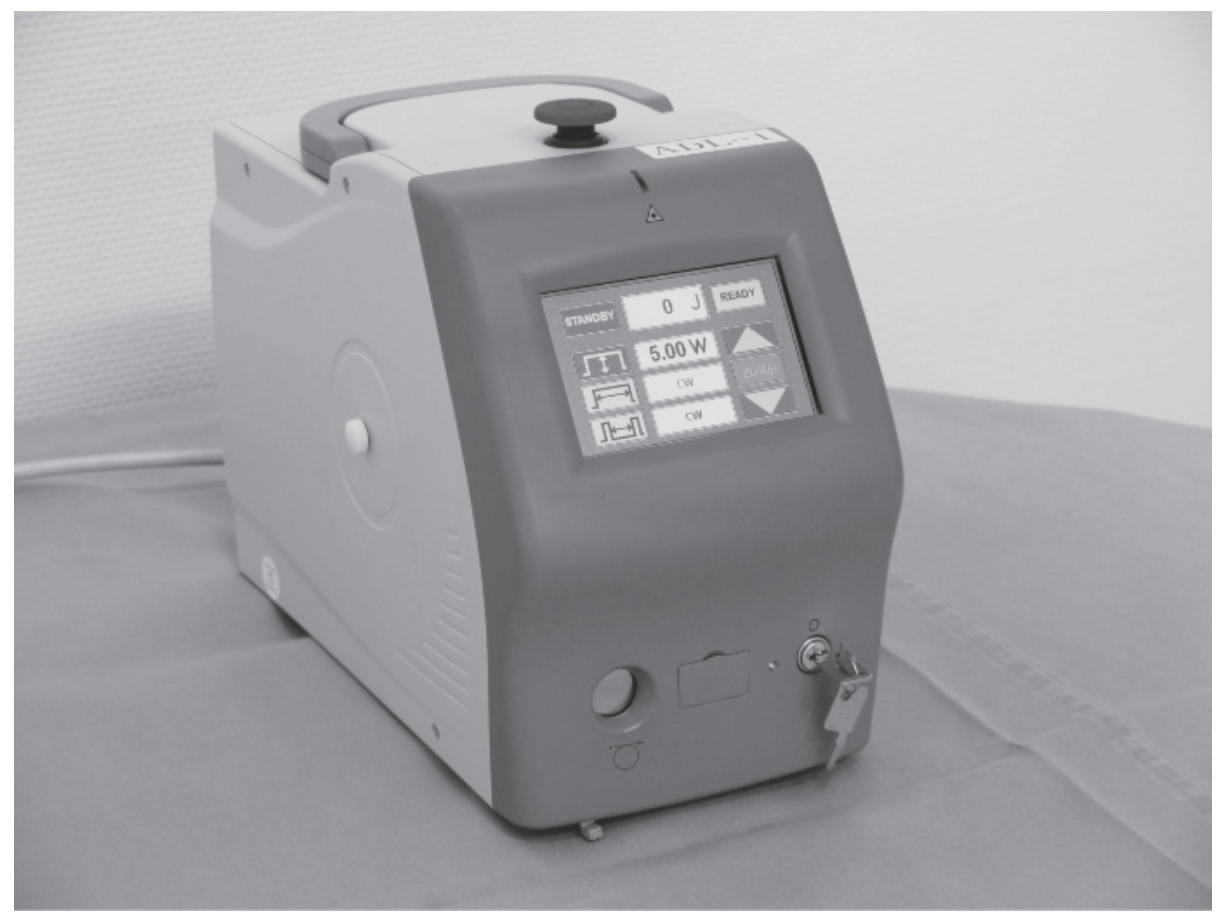

Fig. 1: New 808nm Diode laser DVL-20B (ASUKA Medical Co., Tokyo, Japan) 
$240 \mathrm{~J}$. (Figure 1) Following initial examination patients were seated in a surgical chair and were anesthetized with $8 \%$ lidocaine solution sprayed into each inferior turbinate. Treatment commenced immediately following anesthesia. Treatment at $8 \mathrm{~W}$ was given at irradiation times of $0.5 \mathrm{~s}$, with a 0.5 second interval to a total irradiated energy of $240 \mathrm{~J}$. Laser treatments was delivered to each inferior turbinate with an intranasal probe through an optical fiber. The power density was $41 \mathrm{~J} / \mathrm{cm}^{2}$ at the mucosal lining of the inferior turbinate. Patients returned to the clinic 3 to 4 weeks after treatment for assessment. Subjective assessment from patients was performed according to the symptom score of the Practical Guidelines for Management of Allergic Rhinitis in Japan (PG-MARJ) ${ }^{3)}$. The overall categories are Worse, No change, Improved, Significantly improved and Disappeared.

\section{Results}

Of the 1210 patients treated with the new diode laser, 531 patients were available for follow up and divided into two groups as stated above. In the pre-seasonal group, the average level of pollen allergy was 3.173, and the average result of the immunoglobulin $\mathrm{E}$ radioallergosorbent test (IgE-RAST) was $18.05 \mathrm{UA} / \mathrm{ml}$. On the other hand, in the intra-seasonal group, the average level of pollen allergy was 3.417, and the average IgE-RAST level was $23.85 \mathrm{UA} / \mathrm{ml}$. There was a significant difference between the average IgE-RAST levels between the two groups. $(\mathrm{p}<0.01)$ (Figure 2) Fortyfour point three percent of the patients had other allergies such as house dust or ticks in the pre-seasonal group, with $43.8 \%$ such patients in the intra-seasonal group.

The overall effective ratio was determined as the sum of the patients who scored their treatment as extremely effective ('Disappeared') and effective ('Significantly improved') divided by the total number of patients treated, expressed as a percentage.

Concerning the overall efficacy rates of the treatment for rhinorrhea were $55.6 \%$ and 57.5 in the preseasonal and intra-seasonal groups, with no significant difference between two groups. As for sneezing, the rates were $62.7 \%$ and $54.9 \%$, for nasal obstruction, they were $60.5 \%$ and $66.2 \%$, and for QOL, they were $58.2 \%$

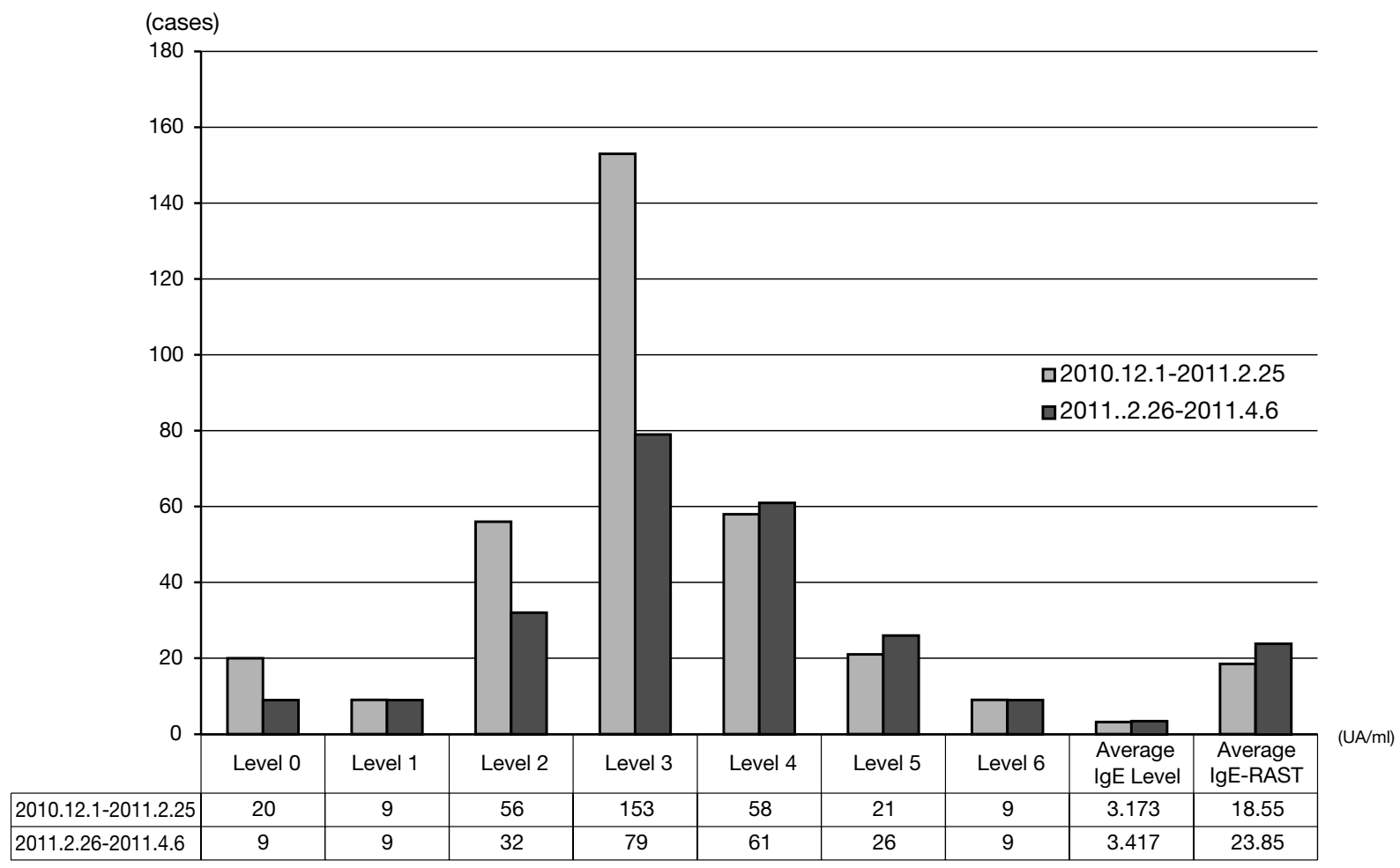

Fig. 2: IgE levels, the average IgE level and average IgE-RAST levels compared between the pre- and intra-seasonal groups. 
and $47.2 \%$ for the pre- and intra-seasonal groups, respectively. Figure 3 summarizes the overall efficacy rates for all symptoms in both groups over the entire study period. Almost $65 \%$ of the patients reported improvement in nasal obstruction, with no significant differences between the two groups.

Figures 4 and 5 show the results of the global efficacy evaluations based on the JP-MARJ categories for the pre- and intra-seasonal groups, respectively. For the nasal obstruction, the intra-seasonal treatment improvement rate was 10\% lower compared to the Preseasonal treatment improvement rate. For rhinorrhea, sneezing, and QOL, there was no significant difference between the two groups in the present study using the $808 \mathrm{~nm}$ diode laser.

After 1 month, 32 patients needed to be treated again, including 29 patients (9.1\%) in the pre-season group and 3 patients (1.3\%) in the intra-season group. There were no cases in which symptoms were exacerbated and no side effects requiring medical attention was observed. On the other hand, the efficacy in prevention of onset of nasal symptoms is shown in Figure 6. All symptoms were been blocked up to almost $70 \%$ in globally over all the patients.
The visible reaction following diode laser treatment consisted of rhinorrhea. We informed all patients that through the severity may differ from patient to patient, rhinorrhea will appear as early as 1 hour following treatment and last for as long as 1 week with the most severe rhinorrhea seen over for the first 2 to 3 days. ${ }^{4)}$

\section{Discussion}

In this present study, there was no significant difference of the treatment efficacy between $808 \mathrm{~nm}$ diode laser treatment for allergic rhinitis between the preand intra-season groups. Generally speaking, intra-season laser treatment has traditionally been avoided because of severe side effects. In 1980-1990, the goal of the $\mathrm{CO} 2$ laser treatment was to either ablate or char the nasal mucosal lining of the inferior turbinate which resulted in complete destruction of mucosal structure. 1) Following a month-long healing process, the mucosa was replaced by scar tissue and hence the mucosa was unable to react to allergic stimuli. The patient had to withstand roughly 2 weeks of complete nasal obstruction and rhinorrhea, and another 2 weeks of crust

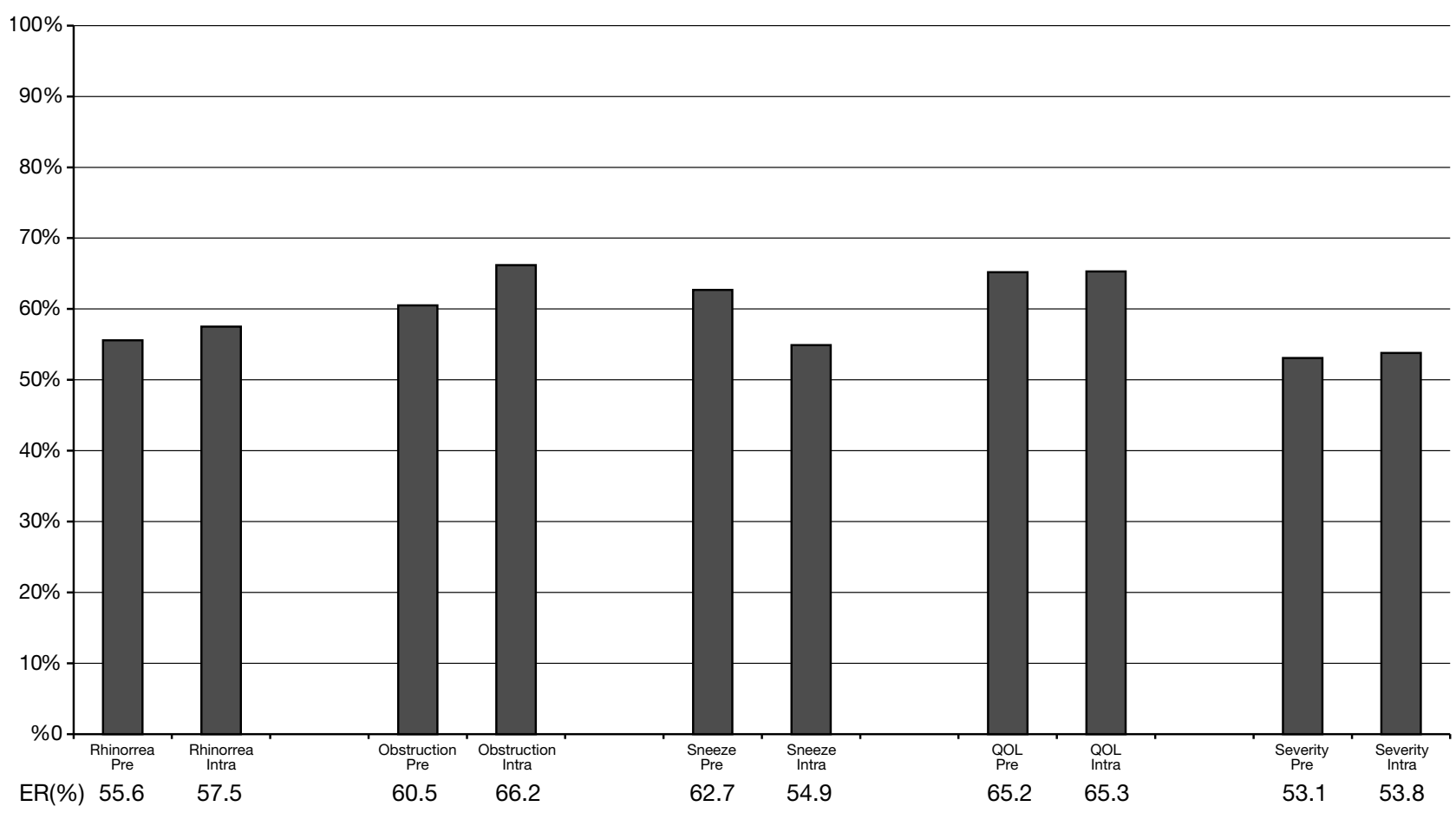

Fig. 3: The overall efficiency ratio (ER) compared between the two study groups -pre and -intra) for all symptoms and overall severity. 


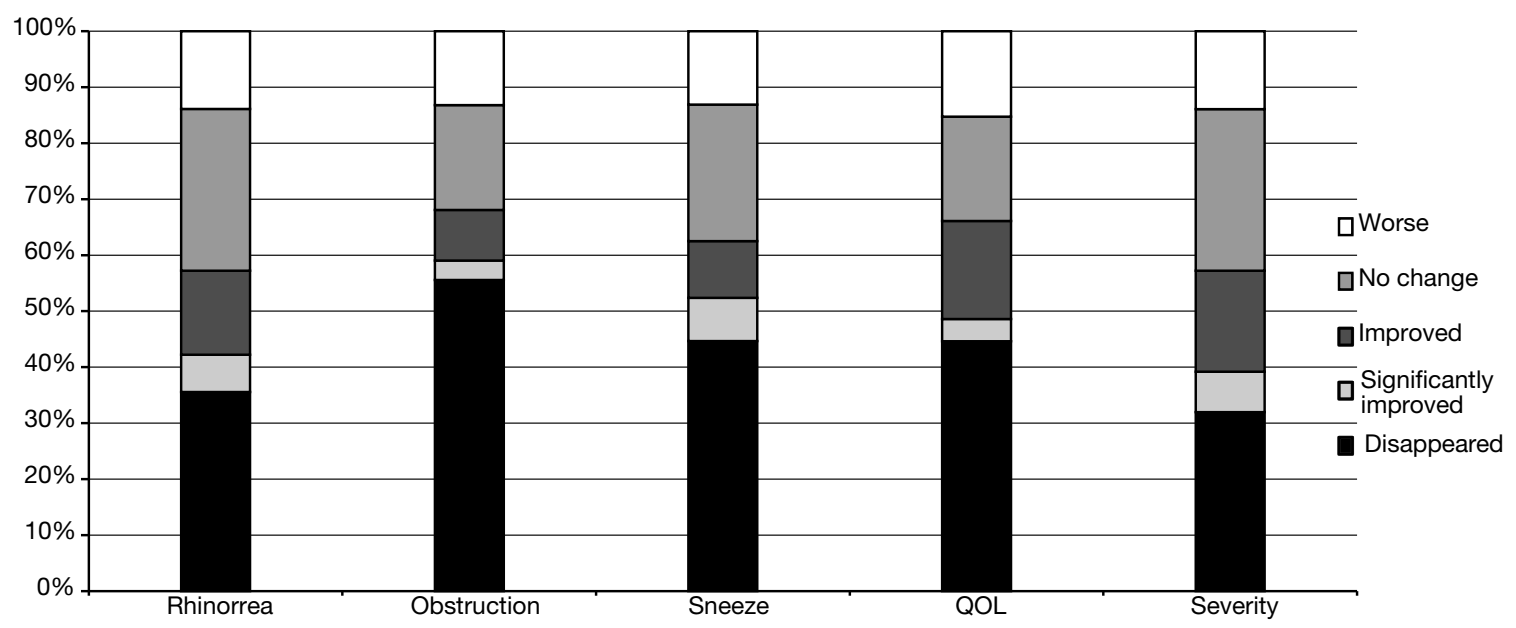

Fig. 4: Treatment results graded by the Practical Guidelines for Management of Allergic Rhinitis in Japan (PG-MARJ) categories for all symptoms and overall severity in the pre-seasonal treatment group.

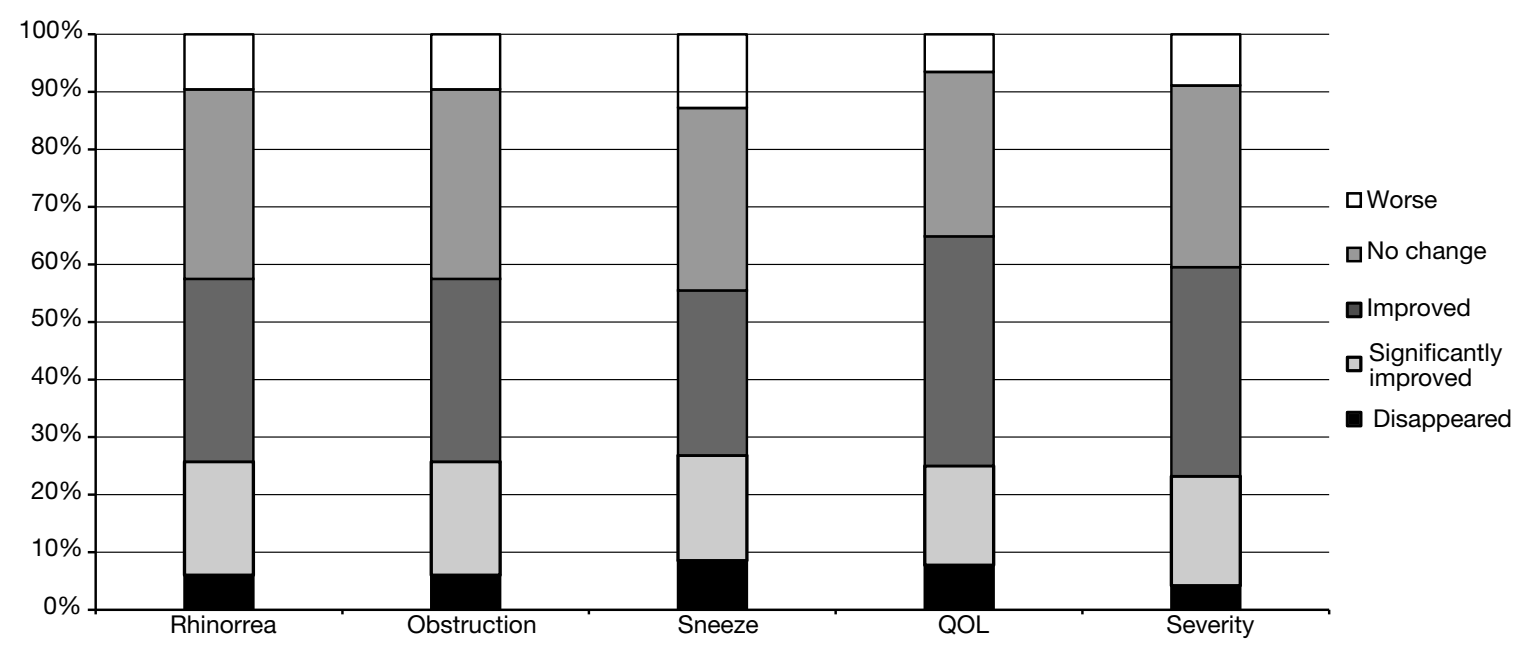

Fig. 5: Treatment results graded by the Practical Guidelines for Management of Allergic Rhinitis in Japan (PG-MARJ) categories for all symptoms and overall severity in the intra-seasonal treatment group.

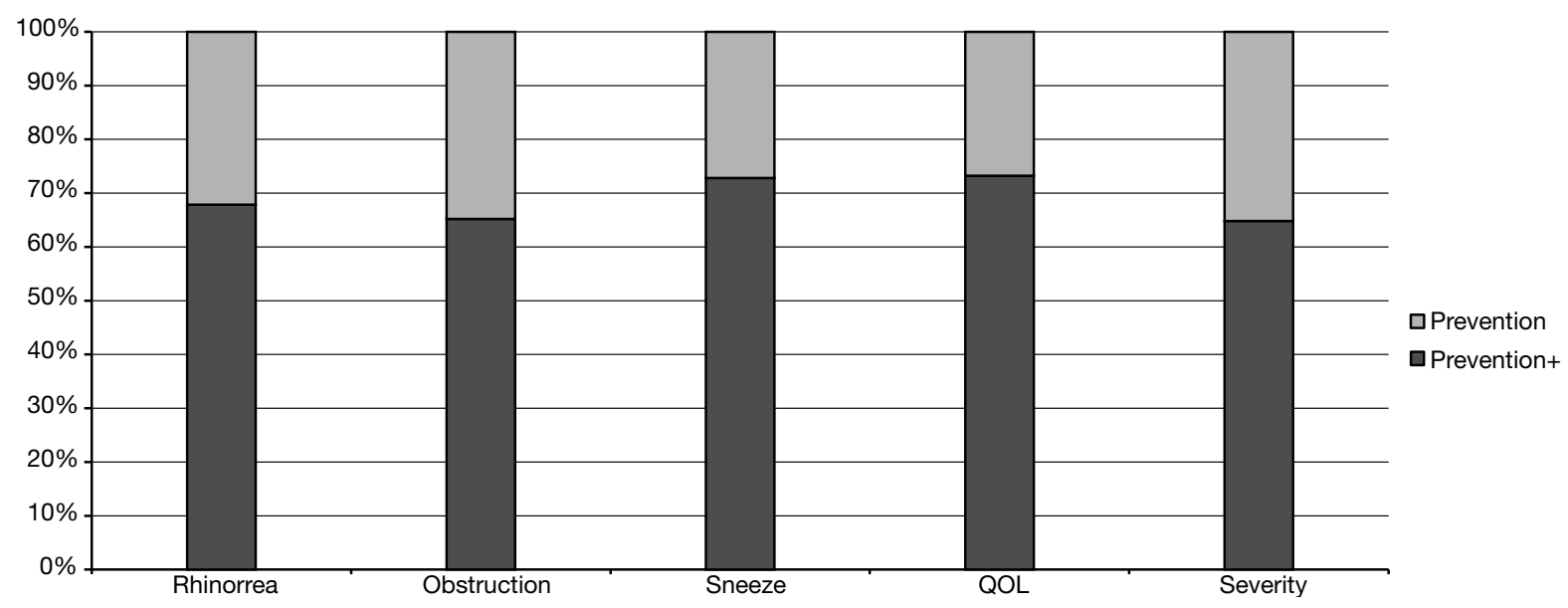

Fig. 6: The overall efficacy in prevention of the onset of nasal symptoms with the $808 \mathrm{~nm}$ diode laser treatment in the 2010-2011 allergic rhinitis seasons. 
removal and occasional nasal bleeding and tenderness. The patient would only be aware of the effect of the treatment after the one month healing process, and if the patient had only seasonal allergic rhinitis. Concerning $\mathrm{CO}_{2}$ laser treatment for pollinosis, the treatment must be performed well before the onset of symptoms. $\left.{ }^{5}, 6\right)$ Despite the side effects mentioned, the $\mathrm{CO} 2$ laser showed promising results ranging from $60 \%$ to as high as $85 \%$ efficacy in the prevention of onset of nasal symptoms. ${ }^{7,8)}$

The flashlamp-pumped Nd: YAG laser also showed promising results ranging from $70 \%$ to as high as $90 \%$ efficacy in the prevention of onset of nasal symptoms. ${ }^{9-11)}$ At the Ohshiro Clinic, we have been using the flash lamp-pumped Nd: YAG laser for the treatment of allergic rhinitis since 1993. Since the 2007 season, we started using a diode laser pumped $\mathrm{Nd}$ : YAG laser system which was developed at our clinic's request by FiberTech Co Ltd (Tokyo, Japan). Most recently, since the 2011 season, we have started using the diode laser system described earlier in the Subjects and Methods section.

\section{Reference}

1: Mittelman H. (1982): CO2 laser turbinectomies for chronic, obstructive rhinitis. Lasers Surg Med. 2:2936

2: Fujii S, Ohshiro T, Ohshiro T, and Sasaki K, Taniguchi Y. (2006): Application of diode pumped Nd: YAG laser for allergic rhinitis. Laser Therapy. $16: 17-24$

3: Baba K, Konno A, Takenaka Y et al. (2009): Practical guideline for the management of allergic rhinitis in Japan. 6th edn. Life Science, Tokyo (in Japanese).

4: Taniguchi Y, Ohshiro T, Ohshiro T, Sasaki K, Fujii S. (2008): Cost-effectiveness of different treatment modalities for seasonal allergic rhinitis in Japan comparison of laser treatment with subcutaneous immunotherapy and oral medication-. Laser Therapy. 17:193-197

5: Kawamura S, Fukutake T, Kudo N, Yamashita T, Kumazawa T. (1993): Subjective results of laser surgery for allergic rhinitis. Acta Otolaryngol Suppl. $500: 109-112$

6: Sandhu AS, Temple RH, Timms MS. (2004) Partial Laser turbinectomy: two year outcomes in patients with allergic and non-allergic rhinitis. Rhinology. 42:81-84
The patient may feel occasional pain but this is well tolerable as has been seen in our experience of treating children as young 10 years of age, or less. The treatment using the $808 \mathrm{~nm}$ diode laser is a quick procedure that does not require a surgical setting and is not associated with any risks. ${ }^{12}$ )

The greatest advantage of diode laser treatment is that there is very little post-operative discomfort. Almost 95\% of the patients did not require any additional treatment within one month, and just over $5 \%$ of the patients who were treated from Feb $26^{\text {th }}$ to Apr $6^{\text {th }}$ needed additional treatments.

\section{Conclusions}

Treatment of allergic rhinitis with the $808 \mathrm{~nm}$ diode laser is safe, effective and comfortable for the patients, with excellent latency and only $5 \%$ or so of patients requiring retreatment. Furthermore, there was no significant difference between the efficacy of treatment given before or during the allergic rhinitis peak season.

7: Imamura S, Honda H. (2003): Carbon dioxide laser vaporization of the inferior turbinate for allergic rhinitis: short-term results. Ann Otol Rhinol Laryngol. 112:1043-1049

8: Chhabra N, Houser SM. (2011): The surgical management of allergic rhinitis. Otolaryngol Clin North Am. 44:779-795

9: Ito H, Suzuki M, Mamiya S, Kitao S, Takagi I, and Baba S. (1995): Clinical effect of Nd: YAG laser treatment on the allergic rhinitis. Arerugi.44:93-95

10: Ito H, Baba S, Suzuki M, Mamiya S, Takagi I, and Kim Y, Kitao S. (1996): Severe perennial allergic rhinitis treated with Nd: YAG laser. Acta Otolaryngol Suppl. 525:14-17

11: Ito H, Takagi S, Nakamura Y, Nishimura J, Suzuki M, and Sakai K, Murakami S. (1999): Effectiveness of Nd: YAG laser therapy on Japanese cedar pollinosis. Nippon Jibiinkoka Gakkai Kaiho. 102:12211226

12: Caffier PP, Scherer H, Neumann K, Luck S, and Enzmann H, Haisch A. (2010): Diode laser treatment in therapy -resistant allergic rhinitis: impact on nasal obstruction associated symptoms. Lasers Med Sci. 26:57-67 